\title{
Detection and characterization of extrasolar planets
}

R. Ferlet ${ }^{\mathrm{a}}$

Institut d'Astrophysique de Paris, CNRS/UPMC, 98bis Bd. Arago, 75014 Paris, France

\begin{abstract}
The main methods to detect planets orbiting stars other than our Sun are briefly described, together with their present results. Some characteristics of the known systems are emphasized. Particularly interesting are the transiting exoplanets which allow to reveal their atmospheres and ultimately identify biosignatures.
\end{abstract}

\section{Introduction}

In November 1995 the famous journal "Nature" published an article [1] which put planetary sciences not only as a new hot field in both observational and theoretical astrophysics but also as a topic with a large impact toward the layman. This article was reporting the first discovery of a planet orbiting a star beyond our Sun, namely the solar-type star 51 Pegasi. Nearly four centuries after Giordano Bruno was publicly burnt in Roma partly for having claimed the plurality of worlds, science is able to set up means in order to begin to answer one of the oldest inquiries of mankind: are we alone in the Universe? To date (September 2008), 313 extrasolar planets are known. We shall briefly review the main detection methods, together with the big surprises which arose during these last exciting thirteen years or so, without being exhaustive.

\section{Direct detection}

To make a picture of an extrasolar planet is of course a major goal, but extremely difficult to reach for two main reasons. First, the angular separation between a planet and its parent star as seen from the Earth is very tiny. For instance, Sun-Jupiter at 4 light-years corresponds to 4 $\operatorname{arcsec}\left(\right.$ or $1 / 900^{\circ}$ ) and at 100 light-years, to $0.15^{\prime \prime}$; Sun-Earth at 100 light-years is only $0.03^{\prime \prime}$. Because of the atmospheric turbulence, optical telescopes cannot resolve objects separated by less than about $1^{\prime \prime}$ in standard conditions. The largest telescopes in the best sites which are equipped with the sophisticated adaptive optics technique, aiming at correcting for the atmospheric turbulence, can presently provide a spatial resolution of at best $0.1^{\prime \prime}$. Second, there is a huge contrast between the planet and the star, the latter can be up to 10 billion times brighter than the former. To image an Earth around a Sun at $10 \mathrm{pc}$ (1 parsec is the distance at which the semi-major axis of the Earth orbit - 1 astronomical unit (AU) or 150 million $\mathrm{km}$ - is seen under an angle of $1^{\prime \prime} ; 1 \mathrm{pc}=3.26$ light-years) would be equivalent to image from Paris a glow-worm located at $30 \mathrm{~cm}$ from a lighthouse in Marseille (about $800 \mathrm{~km}$ away)! This is impossible with the present day optical instrumentation. However, in the near infrared the contrast can be reduced to about 10 million, and it is becoming feasible to register the thermal emission of sub-stellar objects (not yet Earth-like planets) around stars (yet much less bright than the Sun) with adaptive optics. As of September 2008, six planets have been imaged in infrared light $[2-6]$.

\footnotetext{
a e-mail: ferlet@iap.fr
}

Article published by EDP Sciences and available at http://www.epj-conferences.org or http://dx.doi.org/10.1140/epjconf/e2009-00927-6 


\section{Indirect detection: Dynamical effects}

A solution to overcome these difficulties is to use the method of Le Verrier for the discovery of Neptune, namely the gravitational perturbation induced by the planet. As a matter of fact, in a star-planet system, it is both the planet and its parent star that are orbiting the center of mass of the system. For instance, the center of mass of the system Sun-Jupiter is only at 0.005 AU from the center of the Sun (just beyond the solar surface), and Jupiter is inducing a small orbital motion of the Sun of about $12 \mathrm{~m} \cdot \mathrm{s}^{-1}$ around this center of mass, with a period of 11.9 years which is the orbital period of Jupiter at a velocity of $13 \mathrm{~km} \cdot \mathrm{s}^{-1}$. We describe the three different ways for detecting these dynamical effects.

\subsection{Astrometry}

An obvious way to detect the small stellar wobble around the center of mass of the star-planet system, is to monitor the star position on the plane of the sky. Some astronomers spent most of their professional life to that goal. But they never succeeded because the angular scale of the stellar motion is extremely small, even at short distances. For example, the solar motion as seen from $10 \mathrm{pc}$ is of the order of $100 \mu \mathrm{as}$. This is yet too small to be reachable with the present day ground-based optical astrometric techniques, but is within reach of interferometric techniques in course of implementation at e.g. the European Very Large Telescope in Chile, or of already programmed space missions like the European Space Agency project GAIA. The detection of an Earth mass planet around a one solar mass star at $100 \mathrm{pc}$ would require an astrometric accuracy better than 30 nas, still a factor several hundreds below the precision foreseen with GAIA.

To summarize, the astrometric detection can be used with all types of stars, gives access to the exact mass of the planet; it is more sensitive to long periods (or large separations) and is inversely proportional to the distance of the star.

\subsection{Timing}

Another way is to make use of the enormous accuracy reachable when measuring time with atomic clocks. Suppose a rapidly rotating star emitting a very focused electromagnetic radio signal, like a lighthouse. The stellar motion induced by a putative planet can be detected by monitoring the arrival times of the signal when it passes in view of the Earth. Such a celestial extremely regular lighthouse is a pulsar (neutron star), radically different and very much less numerous than solar type or cooler stars. As of September 2008, four planets have been detected around two millisecond pulsars, including one planet no more massive than the Moon $[7,8]$ ! The same method can also be applied to some eclipsing binary stars: with "only" a few seconds accuracy on the eclipses time, a planet of about 3 jovian masses has been detected [9].

To summarize, the timing method allows the detection of objects of very small masses and even satellites; it is insensitive to the distance of the system, but it mainly applies to very peculiar stars - pulsars - which are left-over of some supernovae explosions, implying probably a different formation process than planets around solar type stars. As a matter of fact, planetary objects around pulsars were the very first to be discovered in 1992 [7], but pulsars are not standard "burning" stars on the Main Sequence like our Sun!

\subsection{Velocimetry}

Still to detect the small motion of the parent star around the center of mass, the third way makes use of the spectroscopy. If one records the spectrum of the star when it is approaching the observer, the stellar lines will be blueshifted towards shorter wavelengths because of the Doppler-Fizeau effect; on the contrary, when the star is receding from the observer, stellar lines will be redshifted towards longer wavelengths. Then, by reconstructing the stellar radial 
velocity - the velocity component on the line of sight, directly measurable with the spectral lines - curve as a function of time, it is possible to derive an upper limit to the mass of the planet: $\mathrm{m} . \sin \mathrm{i}$ with $\mathrm{i}$ the inclination angle of the system with respect to the line of sight (if the orbital plane of the star-planet system is seen face-on from the Earth, there is no more Doppler-Fizeau effect, thus no possible detection). Also, from the shape of the velocity curve it is possible to infer the eccentricity of the planetary orbit.

The challenge was to reach an accuracy better than $10 \mathrm{~m} \cdot \mathrm{s}^{-1}$ on radial velocity measurements in order to detect Jupiter-mass planets. This has been successfully performed in 1995 [1] by two Swiss astronomers - Michel Mayor and Didier Queloz - who were observing at the $1.93 \mathrm{~m}$ telescope of the CNRS Observatoire de Haute Provence (OHP, near Forcalquier in France) the star 51 Pegasi with the spectrograph Elodie. The semi-amplitude of the radial velocity curve was $59 \mathrm{~m} \cdot \mathrm{s}^{-1}$, leading to a planetary mass (modulo sin i) of 0.47 jovian mass. Beside being the first exoplanet detection around a solar type star, the big surprise was the period of the planet: 4.231 days, which corresponds to a semi-major axis of $0.05 \mathrm{AU}$, to be compared to the period of Jupiter 11.9 years. It has to be noted that the parameters of the system are derived through modelling of a planetary orbit. In order to get the best fit of the velocity curve, one is led in some cases to introduce several planets in the system.

To summarize, the radial velocity method gives access to different orbital parameters, but is limited to stars showing very many spectral lines in order to increase the measurement precision. Fortunately, this is the case of solar type and cooler stars, the most abundant ones in the Galaxy. Most of the known extrasolar planets were discovered with this method. Naturally, at the beginning the method was biased towards short periods planets, but with time one begins to detect planets more comparable to Jupiter in terms of period. It is also biased towards more massive planets which induce larger velocity shifts. In order to detect an Earth around a Sun, an accuracy of the order of few $\mathrm{cm} \cdot \mathrm{s}^{-1}$ would be required, still far beyond the present instrumental capabilities. Furthermore, since lots of photons are necessary to reach the needed accuracy, up to now only stars in the solar neighborhood are monitored. Last, the method has some limitations inherent to the star itself. For instance, photospheric activity related to spots at the stellar surface, chromospheric activity and seismic activity due to some stellar oscillations (e.g. $[10,11]$ ), can very well mimic radial velocity variations. With the increasing instrumental precision, these very common stellar activities are becoming an important issue. Also, confusion cases can arise from stellar blends such as grazing binaries, small stellar companions or eclipsing binaries in a triple stellar system.

It is interesting to add the existence of a specific spectroscopic signature in the curve of the radial velocity variations. In the case of a planet passing in front of its parent star (a transit, as seen from the Earth; see section 4.2), distortions of the stellar line profiles arise which are due to the small fraction of the rotationally Doppler-shifted stellar disk occulted during the transit [12]. This is the so-called Rossiter - McLaughlin effect which has been observed a few times. It allows to further determine the angle between the orbital plane and the stellar equatorial plane, as well as the rotation velocity of the star.

\section{Photometric detections}

Stellar photometry provides two main ways to detect extrasolar planets, one related to a relativistic effect, the other to a simple geometrical configuration.

\subsection{Gravitational microlensing}

In 1936, Einstein computed [13] the gravitational lensing effect caused by the alignment of a far away source star, the observer and a star in between acting as a gravitational lens bending the light coming from the source star. The observer will perceive a ring of light (the so-called Einstein ring) as the source, if the three points (the source, the lens and the observer) are perfectly aligned; otherwise, he will register an amplification of the source light which can be extremely large depending on the perfection of the alignment (a factor of 100 has already been 
observed). Since the three points are moving, the observer will see an increasing amplification followed by a decreasing one, symmetrically with respect to a maximum, kind of a gaussian curve perfectly well calculable if the source and the lens are supposed to be point-like.

If furthermore there is a planet orbiting the lens star, in certain geometrical configurations either caustic effects or an additional amplification superposed to the main one can be produced. The aim is then to follow as precisely as possible the main amplification due to the lens, which lasts weeks or months, in order to record the secondary planetary amplification, which lasts only hours. As of September 2008, eight planets have been detected through microlensing, including the lightest one (apart the non-standard ones discovered around pulsars) of 3.18 Earth masses [14]. Another one of 5.4 Earth masses with a period of 9.6 years could have been the first ever detected cool rocky-icy exoplanet [15].

To summarize, without any photons from the lens system, the microlensing approach can reach terrestrial planets; however, follow-up is impossible today. The probability to have an alignment of the three points is of the order of $10^{-6}$ or less. Therefore, million of stars have to be surveyed, which opens the possibility of statistical studies in distant $(\approx 4 \mathrm{kpc})$ regions of the Galaxy for which the sensitivity of the method is the best; for instance, no more than few percents of $\mathrm{M}$ stars (the most numerous ones) within these regions have jupiters between 1 and $10 \mathrm{AU}$ from the star. Several projects are presently searching for microlensing events in order to alert worldwide networks of photometric telescopes (such as PLANET: Probing Lensing Anomalies NETwork) to precisely register planetary amplifications [16].

\subsection{Occultation or transit}

If the Earth is more or less in the plane of the orbit of an exoplanet around its parent star, it can be possible to observe the passage of the planet in front of the star. Assuming random orientation of the orbit, the probability to observe such a planetary transit is close to the ratio of the stellar radius over the distance between the star and the planet. For instance, for a faraway observer the probability to observe a transit of Mercury in front of the Sun is about $1 \%$ (planetary periods of the order of 100 days), while it drops down to $0.1 \%$ for Jupiter (planetary periods of the order of 10 years).

The small occultation of the stellar light during the transit (which can last few hours) depends of course upon the radii of the star and the planet. For example, the decrease of the solar flux during a Jupiter transit would be of the order of $1 \%$, which is quite easily detectable. The shape of the transit curve depends also upon the homogeneity of the stellar flux over the stellar disk; in general, it is affected (at least within the visible spectral range) by the limb darkening effect which rounds both sides of the transit photometric profile [17].

The first confirmed planetary transit [18] was the transit of the extrasolar planet HD 209458 b, also named Osiris, recorded with a small aperture ground-based telescope in 1999 $(\approx 1.4 \%$ absorption depth), and accurately studied afterwards with the Hubble Space Telescope (HST). The measured radius is 1.4 jovian radius. This hot jupiter has a period of $3.5247542 \pm 0.0000004$ days, or a semi-major axis of 6.7 million km (Mercury is 8.6 times farther from the Sun). It was discovered by radial velocity searches [19] and has a mass of $0.699 \pm 0.007$ jupiter mass. Combining mass and radius, one obtains an average density of $0.34 \mathrm{~g} \cdot \mathrm{cm}^{-3}$. This was the definitive proof that Osiris is indeed a gas giant. As of September 2008, 52 transiting exoplanets are known. Amongst several on-going transit surveys both ground- and space-based, one can mention the CoRoT satellite presently in orbit (since 27 December 2006) which has just announced its fourth transiting planet [20]; with a photometric accuracy better than $10^{-4}$, Earth size planets are within reach.

To summarize, transits provide several parameters but a crucial one - the radius of the planet - which allows thus a better characterization of exoplanets. Beside limb darkening, several other effects can be observed in the light curve such as: planetary phases; gravitational modulation due to the stellar shape; activity induced by stellar spots; presence of satellites, rings or companions (through shape and timing of transits). 


\section{Exoplanetary systems}

As of September 2008, 313 extrasolar planets are known in 269 planetary systems; 31 of these systems are multiple (more than one planet). Among the 313 exoplanets, 294 have been detected by radial velocity; 52 of them are transiting their parent star; 8 were detected through microlensing; 6 by direct imagery, and 5 by timing (an extrasolar planets encyclopaedia can be found at the URL: www.exoplanet.eu). If one extrapolates the present overall results to the whole Milky Way, one can conclude that several hundreds of million of stars have hot jovian planets orbiting their star in 5 or fewer Earth days.

\subsection{Some properties}

The mass distribution of the 313 exoplanets peaks around 1 jupiter mass. Although, because of an initial instrumental bias, about $20 \%$ or so are hot and very hot jupiters, longer periods more comparable to those in our solar System are more and more common, the number of planets increasing with period (for periods $>100$ days). As a matter of fact, there is an extreme diversity of the orbital parameters (period, semi-major axis, eccentricity). Nevertheless, some possible trends seem to be present: fewer massive planets ( $>2$ jovian masses) at short periods (<100 days); fewer planets between 10 and 100 days; fewer planets less massive than Jupiter at periods $>100$ days; some accumulation of planets at short periods (3-10 days). Contrary to the solar System, extreme eccentricities are known (up to 0.93), yet unexplained.

Another particular, poorly understood, fact is concerning the host stars of extrasolar systems. The distribution of planets as a function of the metallicity (abundance of elements heavier than hydrogen and helium) of host stars peaks at positive values, i.e. stars harboring planets have, on average, a metal content higher than the one found in stars without detected companions. This planet-metallicity correlation seems consistent [21] with the conventional giant planet formation scenario: protoplanetary core accretion (up to roughly 10 Earth masses) followed by gas accretion onto the core.

\subsection{The mass-radius relation}

Knowing both planet sizes provided by photometric transit measurements and planet masses given by e.g. radial velocities, one has access to information on the interior structure of the planets, and ultimately on their formation process. The latest "observational" mass-radius plot for known transiting planets can be found in [22]. The measured sizes are roughly in agreement with theoretical predictions, but there is some scatter, in particular Osiris which has a larger radius and thus seems to be anomalous. Indeed, the recent identification of a star with a planetary size indicates we are on the way to link the mass-radius relation for giant planets and the one for stars. Nevertheless, the planetary and stellar formation processes are radically different.

\subsection{Secondary transit}

For transiting planets, the light emitted by the planets themselves can be identified during the secondary transit, i.e. when planets are passing behind their stars (as seen from the Earth), in the opposite location of the primary eclipse. The flux of the system is coming from both the star and the planet; when the planet is hidden by the star, the observed flux is from the star alone; by substraction, one records the planetary thermal emission. This has been done for few hot jupiters in three different infrared bands by the Spitzer Space Telescope. It is then possible to derive the effective temperature of the planet and its albedo. A temperature above $1000 \mathrm{~K}$ is deduced, even $1150 \mathrm{~K}$ for Osiris [23-25]. Furthermore, the day/night modulation of the infrared $(24 \mu \mathrm{m})$ brightness of one exoplanet has been recorded [26]. 


\section{Spectroscopic transits}

A most promising technique is transmission spectroscopy, i.e. identification of planetary atmospheric signatures as additional absorptions in the stellar spectrum during a transit. The planet will appear larger when observed at a wavelength strongly absorbed by its atmosphere.

\subsection{Osiris}

The first detection of an extrasolar planet atmosphere was performed soon after the discovery of the transit of Osiris: using very high precision spectro-photometric HST data, an additional absorption of $(2.32 \pm 0.57) 10^{-4}$ in the neutral sodium doublet at $589 \mathrm{~nm}$ was measured [27], less than predicted. Still with HST, the Osiris atmosphere was also detected in three other species: neutral hydrogen and oxygen, and singly ionized carbon, against stellar emission lines in the far-ultraviolet $[28,29]$. The first surprise came from the additional absorption measured in HI: $15 \pm 4 \%$, which corresponds to an hydrogen cloud more extended than the Roche lobe of the planet (a cloud with a Roche lobe size would have produced $10 \%$ absorption depth). In this extended exosphere, HI is seen at radial velocities exceeding $100 \mathrm{~km} \cdot \mathrm{s}^{-1}$, because of the radiation pressure acting on the HI atoms. Both facts - atoms beyond the Roche lobe and beyond the planetary escape velocity of $54 \mathrm{~km} \cdot \mathrm{s}^{-1}$ - prove that hydrogen is escaping the planet [28]. Osiris is thus loosing mass!

In order to produce such a large transit signature, numerical simulations show that a massloss rate of at least $10^{10} \mathrm{~g} \cdot \mathrm{s}^{-1}$ is needed. This is in agreement with theoretical modelling of the upper atmosphere structure when taking into account tidal forces and heating by UV and extreme-UV radiations [30]. But another surprise was the detection of OI and CII also in the upper atmosphere, producing about $10 \%$ additional absorption. The mere presence of these heavier elements indicates that the atmosphere is not under the regime of Jeans escape, but is hydrodynamically escaping [29]. Through the evaluation of the total density at the level of the Roche lobe $\left(\approx 10^{6} \mathrm{~cm}^{-3}\right)$, the escape rate can be independently estimated around $10^{10} \mathrm{~g} \cdot \mathrm{s}^{-1}$, in agreement with the value derived from the HI observations.

More recent observations, again with HST, have revealed the presence of several layers of sodium [31], the Rayleigh diffusion by molecular hydrogen [32] and very likely the presence of vanadium and titanium oxides [33]. Sodium is less abundant at high altitude because of its ionization by the stellar radiations or its condensation into molecules. This is the first time that variations in composition are detected in an exoplanet. Thanks to the Rayleigh diffusion, temperature and pressure profiles as a function of altitude have been obtained. At low altitude, temperature should be above $1700^{\circ} \mathrm{C}$; then it seems to decrease below $500{ }^{\circ} \mathrm{C}$, allowing thus sodium condensation; in a higher stratospheric layer, temperature increases again. Last, again through transmission spectroscopy in the infrared with Spitzer, molecules are actively searched for; in particular, water and methane seems to have been identified in the atmosphere of a transiting exoplanet other than Osiris [34].

\subsection{Evaporation of hot jupiters}

The evaporation phenomenon can modify the evolution of hot jupiters. From the computation of the escape rate as a function of mass and orbital period [30], it turns out that even very hot jupiters can be stable against evaporation if they are massive enough. However, those with a period shorter than about three days and less massive than about 0.5 jovian mass can be substantially modified by evaporation. If true, most of the planetary atmosphere should disappear on a timescale shorter than the star life time, leaving a remaining rocky central core of roughly 10 Earth masses, perhaps with a "boiling" lava surface similar to the one of Io in the solar System. These putative bodies, remnants from evaporated hot jupiters, might form a new class of planets, still to be observed. It is interesting to note that a few exoplanets are exactly at the mass-period location predicted for evaporation-modified planets. 


\section{Concluding remarks}

The extrasolar planets field of research is moving forward extremely rapidly, both observationally and theoretically. The number of exoplanets newly detected every year is steadily increasing with both the instrumental accuracy improvements and the number of teams involved worldwide. The extrasolar planets encyclopaedia is recording not less than 77 ground-based extrasolar planets global searches in the world (both ongoing programmes and futures projects) and 19 space-based searches!

Presently, the radial velocity method is the most efficient one thanks mainly to the HARPS spectrograph attached to the European Southern Observatory $3.6 \mathrm{~m}$ telescope in Chile [35], which can achieve a precision better than $1 \mathrm{~m} \cdot \mathrm{s}^{-1}$ (a kind of a clone of HARPS, SOPHIE [36], is installed at OHP, at a $1.9 \mathrm{~m}$ telescope). As a consequence, exoplanets around Neptune's mass are routinely detectable. But of course, the quest for even lower masses is competitively ongoing. For example, a hot Neptune and two super-Earth planets have been discovered with HARPS around a M-dwarf star (much cooler than the Sun) [37]; the lightest one - 5 Earth masses has a period of 12.9 days ( $0.073 \mathrm{AU}$ from the star) and is located within the habitable zone of its parent star, the zone where water could be liquid at the planet's surface (if it is present!).

Photometric methods have the potential of detecting Earth-like planets, and Dome $\mathrm{C}$ in Antarctica is a promising site. However, it is the planetary transits which now offer a first direct vision of extrasolar planets, because of their unprecedented capabilities to probe the characteristics and environment of these planets. But in all cases, the challenge is similar: the photon flux, either from the planet or perturbed by the planetary atmosphere during a transit, is very small. The ultimate goal being to identify bio-signatures in extrasolar planets, larger ground- and space-based telescopes will be mandatory.

The author is very grateful to F. Bouchy for providing material.

\section{References}

1. M. Mayor, D. Queloz, Nature 378, 355 (1995)

2. G. Chauvin, et al., Astron. Astrophys. 425, L29 (2004)

3. R. Neuhäuser, et al., Astron. Astrophys. 435, L13 (2005)

4. G. Chauvin, et al., Astron. Astrophys. 438, L29 (2005)

5. B.A. Biller, et al., Astrophys. J. 641, L141 (2006)

6. V. Béjar, et al., Astrophys. J. 673, L185 (2008)

7. A. Wolszczan, D. Frail, Nature 355, 145 (1992)

8. D. Backer, et al., Nature 365, 817 (1993)

9. R. Silvotti, et al., Nature 449, 189 (2007)

10. N. Santos, et al., Astron. Astrophys. 361, 265 (2000)

11. M. Bazot, et al., Astron. Astrophys. 470, 295 (2007)

12. D. Queloz, et al., Astron. Astrophys. 359, L13 (2000)

13. A. Einstein, Science 84, 506 (1936)

14. D. Bennett, et al., Astrophys. J. 684, 663 (2008)

15. D. Ehrenreich, et al., Astrophys. J. 651, 535 (2006)

16. M. Dominik, et al., Planet. Space Sci. 50, 299 (2002)

17. T. Brown, et al., Astrophys. J. 552, 699 (2001)

18. D. Charbonneau, et al., Astrophys. J. 529, L45 (2000)

19. T. Mazeh, et al., Astrophys. J. 532, L55 (2000)

20. S. Aigrain, et al., Astron. Astrophys. 488, L43 (2008)

21. J. Valenti, D. Fischer, Phys. Scr. 130, 014003 (2008)

22. F. Pont, et al., Astron. Astrophys. 487, 749 (2008)

23. D. Charbonneau, et al., Astrophys. J. 626, 523 (2005)

24. D. Deming, et al., Nature 434, 740 (2005)

25. D. Deming, et al., Astrophys. J. 644, 560 (2006)

26. J. Harrington, et al., Science 314, 623 (2006)

27. D. Charbonneau, et al., Astrophys. J. 568, 377 (2002) 
28. A. Vidal-Madjar, et al., Nature 422, 143 (2003)

29. A. Vidal-Madjar, et al., Astrophys. J. 604, L69 (2004)

30. A. Lecavelier des Etangs, et al., Astron. Astrophys. 418, L1 (2004)

31. D. Sing, et al., Astrophys. J. 686, 667 (2008)

32. A. Lecavelier des Etangs, et al., Astron. Astrophys. 485, 865 (2008)

33. J.-M. Désert, et al., Astron. Astrophys. 492, 585 (2008)

34. M. Swain, et al., Nature 452, 329 (2008)

35. M. Mayor, et al., The ESO Messenger 114, 20 (2003)

36. F. Bouchy, et al., Tenth Anniversary of 51 Peg-b: Status of and prospects for hot Jupiter studies (Frontier Group, OHP, 2006), p. 319

37. S. Udry, et al., Astron. Astrophys. 469, L43 (2007) 